\title{
Metabolic changes associated with adaptive resistance to daptomycin in Streptococcus mitis-oralis
}

\author{
Allison Parrett', Joseph M. Reed ${ }^{2,3}$, Stewart G. Gardner ${ }^{2,4}$, Nagendra N. Mishra, ${ }^{5,6}$, Arnold S. Bayer ${ }^{5,6}$,
} Robert Powers ${ }^{1,7^{*}}$ and Greg A. Somerville $e^{2^{*}}$ (D)

\begin{abstract}
Background: Viridans group streptococci of the Streptococcus mitis-oralis subgroup are important endovascular pathogens. They can rapidly develop high-level and durable non-susceptibility to daptomycin both in vitro and in vivo upon exposure to daptomycin. Two consistent genetic adaptations associated with this phenotype (i.e., mutations in cdsA and pgsA) lead to the depletion of the phospholipids, phosphatidylglycerol and cardiolipin, from the bacterial membrane. Such alterations in phospholipid biosynthesis will modify carbon flow and change the bacterial metabolic status. To determine the metabolic differences between daptomycin-susceptible and nonsusceptible bacteria, the physiology and metabolomes of S. mitis-oralis strains 351 (daptomycin-susceptible) and 351-D10 (daptomycin non-susceptible) were analyzed. S. mitis-oralis strain 351-D10 was made daptomycin nonsusceptible through serial passage in the presence of daptomycin.

Results: Daptomycin non-susceptible S. mitis-oralis had significant alterations in glucose catabolism and a rebalancing of the redox status through amino acid biosynthesis relative to daptomycin susceptible S. mitis-oralis. These changes were accompanied by a reduced capacity to generate biomass, creating a fitness cost in exchange for daptomycin non-susceptibility.

Conclusions: S. mitis-oralis metabolism is altered in daptomycin non-susceptible bacteria relative to the daptomycin susceptible parent strain. As demonstrated in Staphylococcus aureus, inhibiting the metabolic changes that facilitate the transition from a daptomycin susceptible state to a non-susceptible one, inhibits daptomycin nonsusceptibility. By preventing these metabolic adaptations in S. mitis-oralis, it should be possible to deter the formation of daptomycin non-susceptibility.
\end{abstract}

Keywords: Streptococcus, Metabolism, Antibiotic resistance, Daptomycin

\footnotetext{
*Correspondence: rpowers3@unl.edu; gsomerville3@unl.edu

'Department of Chemistry, University of Nebraska-Lincoln, Lincoln, NE 68588-0304, USA

${ }^{2} \mathrm{School}$ of Veterinary Medicine and Biomedical Sciences, University of Nebraska-Lincoln, Lincoln, NE 68588-0905, USA

Full list of author information is available at the end of the article
}

(c) The Author(s). 2020 Open Access This article is licensed under a Creative Commons Attribution 4.0 International License, which permits use, sharing, adaptation, distribution and reproduction in any medium or format, as long as you give appropriate credit to the original author(s) and the source, provide a link to the Creative Commons licence, and indicate if changes were made. The images or other third party material in this article are included in the article's Creative Commons licence, unless indicated otherwise in a credit line to the material. If material is not included in the article's Creative Commons licence and your intended use is not permitted by statutory regulation or exceeds the permitted use, you will need to obtain permission directly from the copyright holder. To view a copy of this licence, visit http://creativecommons.org/licenses/by/4.0/ The Creative Commons Public Domain Dedication waiver (http://creativecommons.org/publicdomain/zero/1.0/) applies to the data made available in this article, unless otherwise stated in a credit line to the data. 


\section{Background}

Staphylococcus aureus is the most common cause of infective endocarditis (IE) in the industrialized world [1, 2]. The viridans group streptococci are the second leading IE pathogen world-wide, and the most frequent etiology of IE in developing countries. Among the viridans group streptococci, the $S$. mitis-oralis subgroup (i.e., $S$. mitis, S. oralis, S. gordonii and S. parasanguinis) are the predominant IE etiologies. This sub-group is therapeutically problematic, as between 10 and $40 \%$ of strains exhibit resistance to penicillins and/or cephalosporins, including ceftriaxone $[3,4]$. For this reason, the majority of research into $S$. mitis-oralis antibiotic resistance todate relates to the genetic determinants of penicillin resistance (e.g., [5]).

In addition to being multi- $\beta$-lactam-resistant, $S$. mitisoralis can also be vancomycin-tolerant [6], which increases the use of daptomycin in treating infections caused by such bacteria. Importantly, daptomycin nonsusceptibility arises rapidly both in vitro and in vivo (e.g., during the treatment experimental infective endocarditis [7]), causing great concern this could occur in humans undergoing daptomycin therapy for streptococcal IE. In S. mitis-oralis, daptomycin non-susceptibility is associated with mutations in $c d s A$ and $\operatorname{pgs} A[8,9]$, genes involved in biosynthesis of membrane phospholipids [10]. Specifically, these mutations in daptomycin non-susceptible $S$. mitis-oralis strains result in the loss of phosphotidylglycerol and cardiolipin from the membrane.

Although these studies have been critical for understanding the genetic perturbations that facilitate $S$. mitisoralis non-susceptibility to daptomycin, the physiologic and metabolic modifications associated with adaptive resistance to daptomycin are unknown. In S. aureus, daptomycin non-susceptibility has been metabolically linked to decreased tricarboxylic acid (TCA) cycle activity, as well as increased nucleotide synthesis and carbon flow to pathways important for wall teichoic acid and peptidoglycan biosynthesis [11]. Because $S$. mitis-oralis lacks a TCA cycle and grows best in a reduced oxygen environment, it is likely the metabolic adaptations underpinning daptomycin non-susceptibility are different from those found in $S$. aureus. To determine the metabolic changes associated with adaptive resistance to daptomycin in S. mitis-oralis, the metabolomes of daptomycinsusceptible strain 351 and it's in vitro-derived daptomycin non-susceptible variant, 351-D10, were analyzed using nuclear magnetic resonance (NMR) spectroscopy.

\section{Results}

Daptomycin non-susceptibility alters growth of S. mitis The transition of $S$. mitis-oralis strain 351 from a daptomycin-susceptible state to a non-susceptible state (strain 351-D10) during serial passage was accompanied by a decreased growth rate (351 and 351-D10; $29 \pm 1$ min and $39 \pm 1.5 \mathrm{~min}$, respectively) and a decreased growth yield/biomass (Fig. 1a and Supplemental Fig. 1). This altered growth phenotype was reflected in the significant differences in the rates of cultivation media acidification (Fig. 1b). As expected, the growth changes also decreased glucose depletion from the medium containing strain 351-D10 relative to that of strain 351 (Fig. 1c and Supplemental Fig. 2). Importantly, the total amount of glucose consumed by both strains was equivalent, yet the difference in biomass between the two strains was significant, suggesting that growth alone did not account for all the differences in glucose consumption. The glycolytic end-product of glucose is pyruvate, which, in $S$. mitis-oralis, is predominantly catabolized by the membrane-associated lactate dehydrogenase [12]. The lactic acid produced by lactate dehydrogenase accumulated in the cultivation media, which caused the $\mathrm{pH}$ of the media to decrease over time (Fig. $1 b$ and d). Similar to the depletion of glucose from the media, the accumulation of lactate in the media was largely dependent on bacterial growth (Fig. 1d and Supplemental Fig. 2). Taken together, the transition of S. mitis-oralis to a daptomycin non-susceptible state was accompanied by significant fitness changes resulting from impaired growth, and, likely, altered metabolism.

\section{The transition to a daptomycin non-susceptible state significantly alters metabolism}

The growth profiling of S. mitis-oralis strains 351 and 351-D10 suggests metabolism was altered during the transition to a daptomycin non-susceptible state. To assess the extent of metabolic alterations, ten independent replicates of cell-free lysates from strains 351 and 351D10 cultivated with ${ }^{13} \mathrm{C}$-glucose were harvested, the $1 \mathrm{D}$ ${ }^{1} \mathrm{H}$ NMR spectra were collected, and the spectra were analyzed by PCA (Fig. 2). To normalize the metabolomic samples for the dissimilar growth kinetics of the two strains, and to ensure the metabolomes represented equivalent growth phases, bacteria were harvested at different cultivation times (i.e., $2 \mathrm{~h}$ for strain 351 and $2 \mathrm{~h} 45 \mathrm{~min}$ for strain 351-D10). As expected, the PCA scores plot revealed that daptomycin susceptible and non-susceptible strains each formed well-separated clusters (Fig. 2), confirming that significant metabolic differences arose during the transition to daptomycin non-susceptibility.

The PCA scores plot established significant metabolic differences existed between daptomycin-susceptible and non-susceptible S. mitis-oralis strains (Fig. 2), and the growth profiles indicated these differences were likely related to the enzymatic processing of glucose (Fig. 1c and d). To assess the differences in glucose catabolism and 


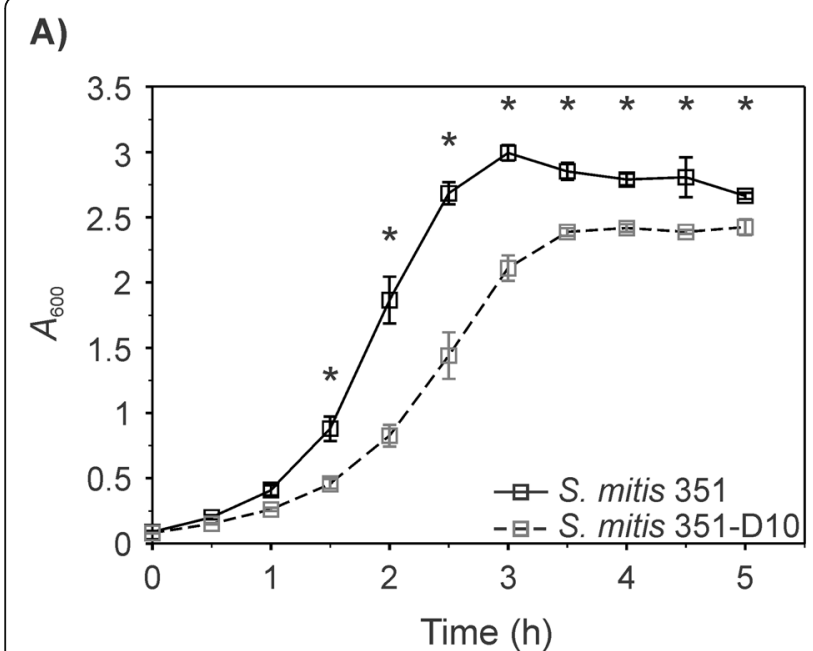

B)

C)
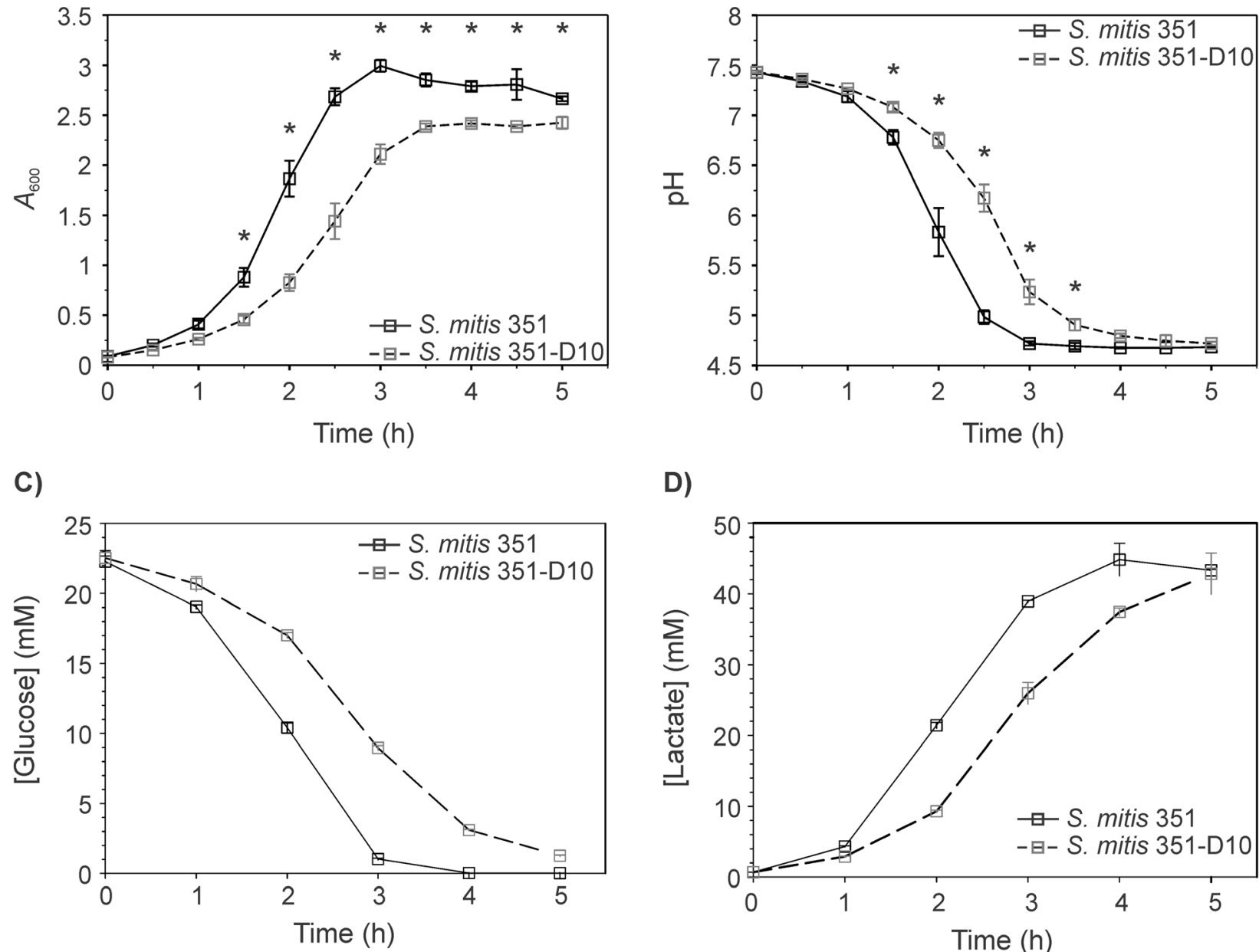

D)

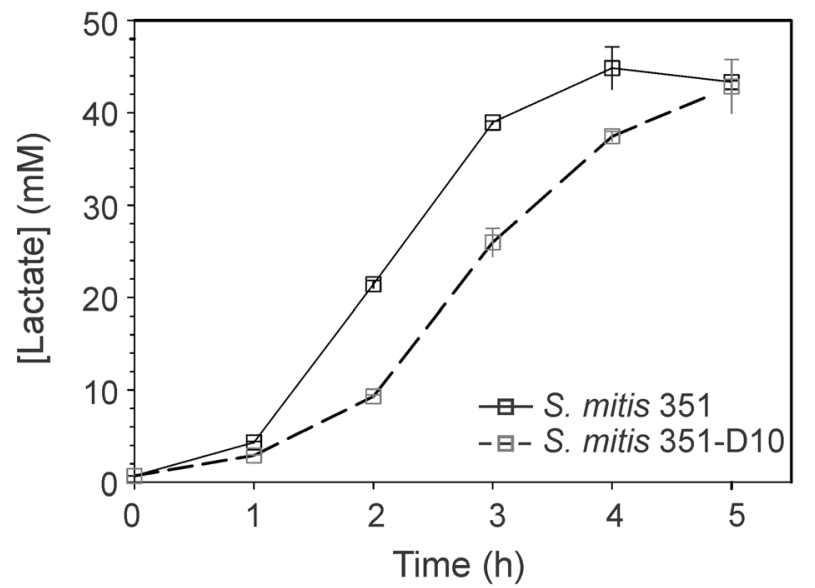

Fig. 1 Growth characteristics of S. mitis-oralis strains 351 (black symbols, solid line) and 351-D10 (grey symbols, dashed line) cultivated in BHI with $2 \mathrm{~g} / \mathrm{L}$ of supplemental glucose. The $A_{600}(\mathbf{a})$ and $\mathrm{pH}(\mathbf{b})$ profiles were assessed every $30 \mathrm{~min}$. Glucose depletion (c) and lactate accumulation (d) in the cultivation media were assessed hourly. Data are representative of the mean of experiments performed in biological triplicate, with error bars representing the standard deviation of the mean. Statistical differences in $A_{600}$ and pH for strain 351 relative to strain 351-D10 was assessed by ANOVA. A statistically significant difference $(p \leq 0.01)$ is represented with an $\left(^{*}\right)$

downstream pathways between strains 351 and 351-D10, the bacterial cell-free lysates used in the PCA analysis were analyzed by collecting $2 \mathrm{D}{ }^{1} \mathrm{H}-{ }^{13} \mathrm{C}$ HSQC NMR (Fig. 3 and Supplemental Fig. 3). Consistent with the growth profiles, S. mitis-oralis strain 351-D10 had significantly decreased intracellular levels of activated glucose (i.e., UDP-glucose) and lactic acid relative to strain 351 (Fig. 3). In contrast, strain 351-D10 had a significantly greater level of acetic acid, indicating that pyruvate generated from glycolysis was used for substratelevel phosphorylation as opposed to almost exclusively being used for the oxidation of NADH via lactate dehydrogenase. This difference was consistent with decreased GAPDH activity in strain 351-D10 (Fig. 4). Decreased GAPDH activity would slow the accumulation of NADH, but also decrease glycolytic ATP synthesis. As mentioned, strain 351-D10 likely offsets the decrease in glycolytic ATP synthesis through substrate phosphorylation via the phosphotransacetylase/ acetate kinase pathway (Fig. 3).

\section{Discussion}

Most studies of antibiotic resistance/non-susceptibility focus on genetic, transcriptional, and occasionally proteomic analyses $[5,8,10]$. These studies can provide valuable information; however, they describe changes that are predicted to occur (e.g., gene transcription does not necessarily equate with protein translation or activity). In contrast, metabolomics provides insight into cellular changes that have actually occurred and are likely to contribute to antibiotic non-susceptibility. Because metabolic pathways are finite in number, it is possible to 


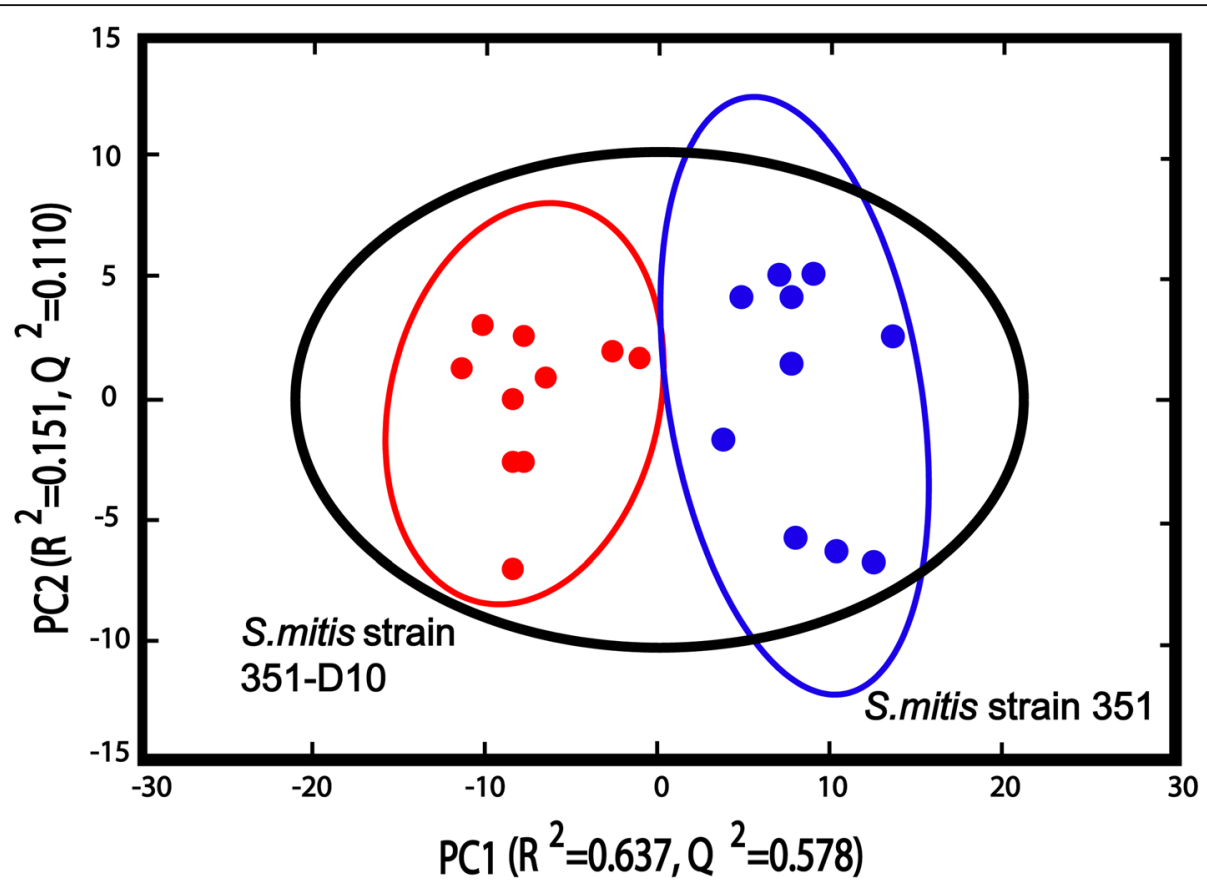

Fig. 2 The PCA scores plot was generated from 1D ${ }^{1} \mathrm{H}$ NMR spectra collected using cell-free lysates of S. mitis-oralis strains 351 (blue) and 351 D10 (red) cultivated in BHI with $2 \mathrm{~g} / \mathrm{L}$ of supplemental ${ }^{13} \mathrm{C}$-glucose and harvested at $2 \mathrm{~h}$ (351) or $2 \mathrm{~h} 45 \mathrm{~min}(351-\mathrm{D} 10)$. The $\mathrm{R}^{2}$ and $\mathrm{Q}^{2}$ are 0.931 and 0.781 respectively for the PCA model

'work backwards' from the profile of altered metabolites and determine where the alterations arose.

In S. aureus, daptomycin non-susceptibility can be mediated by genetic changes in $m p r F$, walKR, rpoB, and rpoC [13-16], changes in cell membrane composition $[17,18]$, and/or metabolic adaptations [11]. The metabolic adaptations in $S$. aureus cause a redirection of carbon flow from the TCA cycle into the pentose phosphate pathway. This redirection of carbon increases the availability of essential intermediates for the biosynthesis of peptidoglycan, wall teichoic acids, and nucleosides/nucleotides. These metabolic changes pre-adapt daptomycin non-susceptible $S$. aureus for daptomycin challenge. In other words, daptomycin challenge causes only minimal metabolic perturbations, which minimizes the fitness cost of daptomycin non-susceptibility [11]. The more likely reason for the minimal metabolic changes and low fitness cost associated with daptomycin non-susceptibility in $S$. aureus is due to its relatively robust metabolic capabilities, including the full complement of central metabolism pathways $[19,20]$. In contrast, S. mitis-oralis lacks the central metabolism TCA cycle, creating metabolic deficiencies that require supplementation from the medium or a host. Alterations in the more modest metabolic capabilities of S. mitisoralis would be consistent with profound growth differences between the susceptible and non-susceptible strains (Fig. 1).
The growth differences between daptomycin susceptible and non-susceptible $S$. mitis-oralis strains are indicative of significant metabolic changes. Metabolomics revealed these growth differences coincided with significant metabolic changes (Fig. 2). Most importantly, is an alteration in how glucose is catabolized (Figs. 1, 3, and 4). For a lactic acid bacterium, alterations in glycolysis and/or lactate dehydrogenase activity creates the problem of how to produce ATP and maintain the redox status. Generating ATP by substrate-level phosphorylation has the advantage of not reducing $\mathrm{NAD}^{+}$, which decreases the burden on lactate dehydrogenase to balance the redox state, although it does not eliminate the need to oxidize dinucleotides. In addition to differences in ATP generation, the altered/delayed glucose catabolism allows for the diversion of carbon into other metabolic pathways (Figs. 3 and 5), which can enable metabolic adaptations that overcome altered glucose utilization. In contrast to $S$. aureus, S. mitis did not shunt carbon into the oxidative branch of the pentose phosphate pathway, which would generate NADPH. This latter point further indicates that these metabolic changes are driven by the bacterial redox status.

In daptomycin non-susceptible S. mitis-oralis, the metabolomics data revealed that glucose carbons were more slowly catabolized through glycolysis and lactate dehydrogenase relative to the daptomycin-susceptible strain (Figs. 1 and 3). As the primary function of lactate 


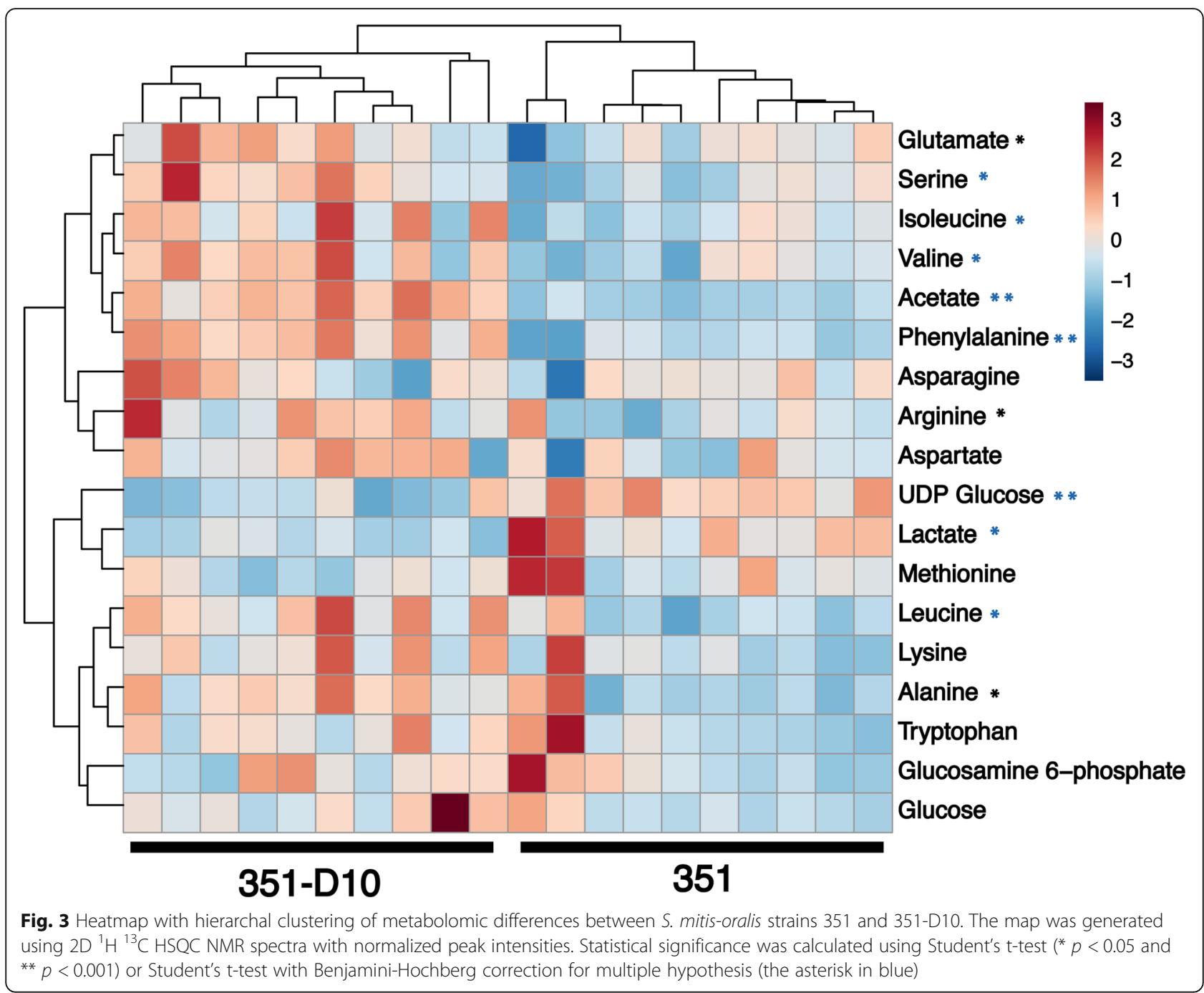

dehydrogenase is maintaining redox balance, it is likely a dysregulation in redox status contributed to the slower growth of strain 351-D10. One possibility for maintaining the redox status is by funneling carbon through amino acid biosynthetic pathways that oxidize more dinucleotides (e.g., branched chain amino acids, glutamate, and alanine) than they reduce (e.g., serine) (Fig. 5). Funneling more carbon through amino acid synthesis can be done in such a way as to facilitate the oxidation of dinucleotides, but the ATP deficit created by decreasing carbon flow through glycolysis also needs to be offset. The increase in acetic acid strongly suggests the ATP deficit is balanced by substrate-level phosphorylation through the phosphotransacetylase/acetate kinase pathway. Taken together, these data suggest the transition of $S$. mitis-oralis to a daptomycin non-susceptible state is accompanied by changes in redox balance, ATP synthesis, and carbon utilization. The extent to which these metabolic changes confer daptomycin non-susceptibility, or are a consequence of mutations arising in phospholipid biosynthetic genes, remains to be determined. In addition, the contribution of each daptomycin-induced mutation to the metabolic changes remains to be determined. Lastly, an important unanswered question is whether these metabolic changes in daptomycin-nonsusceptible $S$. mitis-oralis strains be exploited to prevent or overcome this phenotype, as demonstrated in daptomycin-non-susceptible S. aureus [21, 22]? On this point, metabolic adaptations that facilitate nonsusceptibility to vancomycin and daptomycin have successfully been targeted in $S$. aureus to prevent the development of non-susceptibility and to re-sensitize nonsusceptible bacteria to these antibiotics [21, 22]. This strategy has relied on using inhibitors of metabolic pathways important for non-susceptibility such that enzymatic activity is reduced to the point where it becomes detrimental to the development or maintenance of nonsusceptibility. Similar success with $S$. mitis-oralis will likely target amino acid biosynthesis and/or glycolysis, but this work is on-going. 


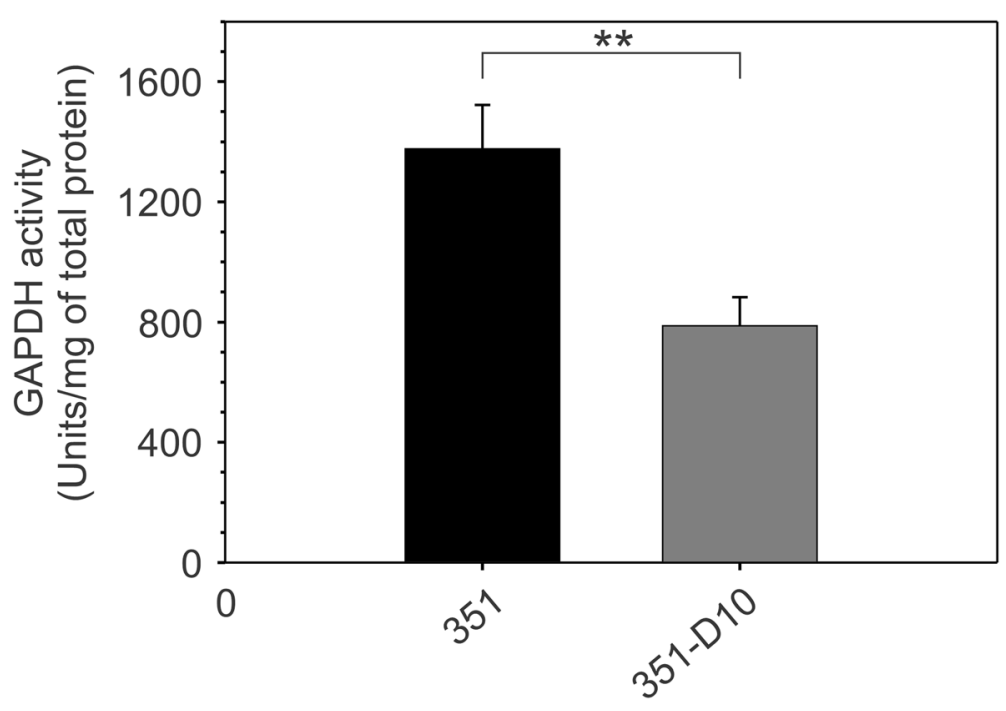

S. mitis Strain

Fig. 4 GAPDH activity in S. mitis-oralis strain 351-D10 is significantly reduced relative to strain 351. GAPDH activity was measured in S. mitis-oralis cell lysates from cultures cultivated in BHI and harvested after $1.5 \mathrm{~h}$ (351) or $2.5 \mathrm{~h}$ (351-D10). The data represent the mean and standard deviation from the mean of 3 biological replicates. Statistical significance $(* *)$ was assessed using Student's t-test $(p \leq 0.05)$

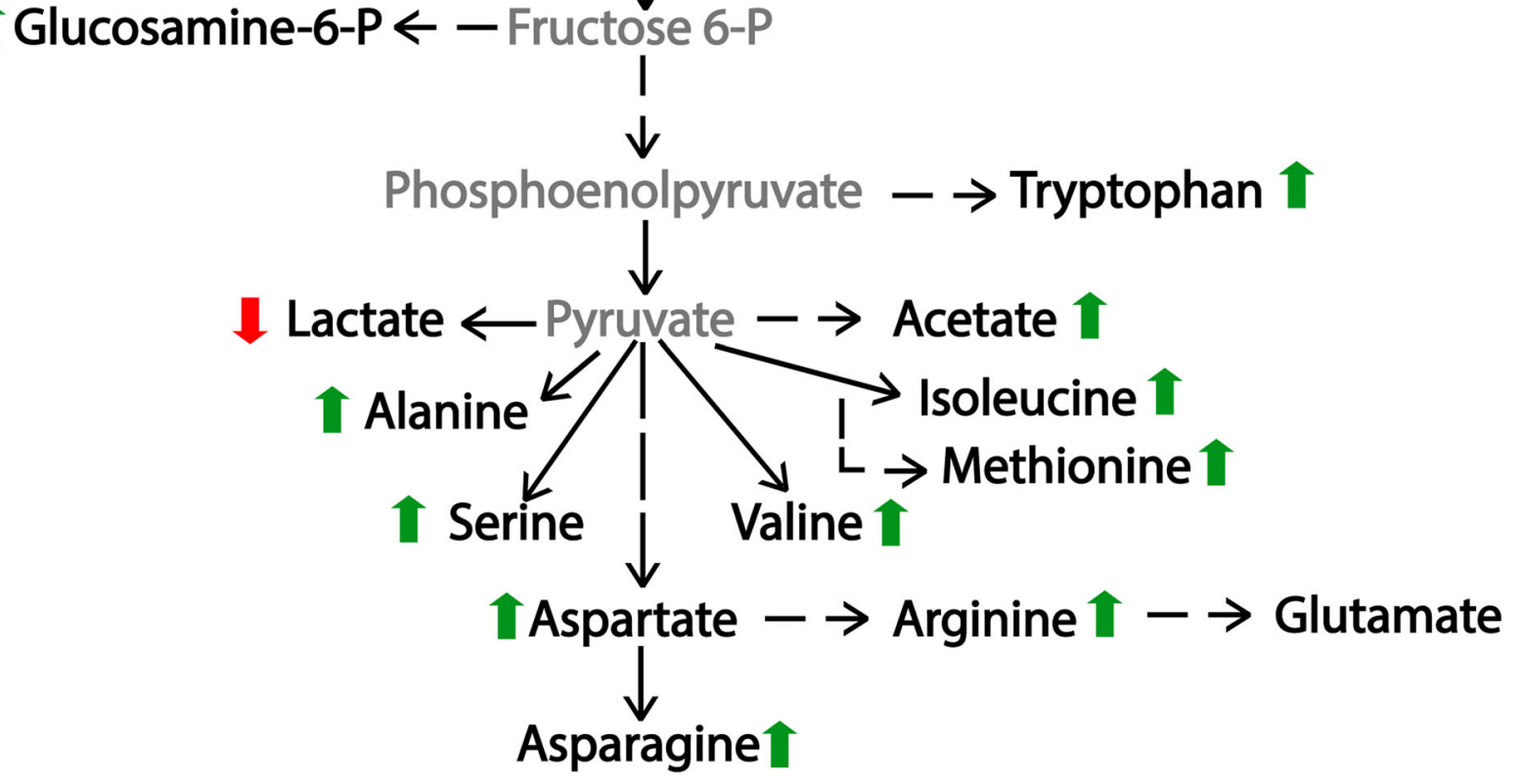

Fig. 5 Summary of metabolic changes between S. mitis-oralis strains 351 and 351-D10. Green upward arrows represent a metabolite whose concentration is increased in strain 351-D10 relative to those in strain 351. Red downward arrows represent metabolites that are decreased in strain 351-D10 relative to that in strain 351. Metabolites in gray represent intermediates that were not observed in the NMR spectra, but were inferred from secondary metabolites 


\section{Conclusions}

Significant metabolic changes accompany the development of a daptomycin non-susceptible state in S. mitis. These changes alter the bacterial ability to utilize glucose. This is a significant observation because $S$. mitis, like most lactic acid bacteria, is heavily dependent upon glycolysis and lactate dehydrogenase to balance the redox state and provide energy and biosynthetic intermediates. Because these changes occur in an essential metabolic pathway, it should be possible to target glycolysis using drugs that increase stress on this pathway (e.g., oxamic acid) and prevent the development of daptomycin non-susceptibility and to re-sensitize nonsusceptible bacteria to daptomycin.

\section{Methods}

\section{Bacterial strains and cultivation conditions}

$S$. mitis-oralis daptomycin-susceptible strain 351 (daptomycin $\mathrm{MIC}=0.5 \mu \mathrm{g} / \mathrm{ml}$ ) and its daptomycin nonsusceptible derivative, 351-D10 (daptomycin MIC > $256 \mu \mathrm{g} / \mathrm{ml}$ ), were used for metabolomic analysis [8]. $S$. mitis-oralis strain 351 was isolated from a patient with endocarditis [7] and the derivative strain 351-D10 was generated by in vitro serial passage of strain 351 for 10 days in medium containing $20 \mu \mathrm{g} / \mathrm{ml}$ of daptomycin [8]. Whole-genome sequencing of the two strains revealed that strain 351-D10 had accrued mutations at seven loci; specifically, orfs sm351-26 (cdsA), sm351-42 (rpoB), sm351-129 (fni), sm351-251 (unknown), sm351-669 (pepT), sm351-1076 (rbn), and sm351-1167 (clpX). S. mitis strains were cultivated in Bacto Brain Heart Infusion medium (BHI), BHI + $2 \mathrm{~g} / \mathrm{L}$ D-Glucose $(\mathrm{BHI}+\mathrm{glu}-$ cose), $\mathrm{BHI}+2 \mathrm{~g} / \mathrm{L}{ }^{13} \mathrm{C}$-D-Glucose $\left(\mathrm{BHI}+{ }^{13} \mathrm{C}\right.$-glucose $)$, or on blood agar (TSA with Sheep Blood). Bacterial precultures were inoculated 1:100 from overnight cultures into $50 \mathrm{~mL}$ of $\mathrm{BHI}$ in a $50 \mathrm{~mL}$ conical tube and incubated at $37^{\circ} \mathrm{C}$, without aeration, for $4 \mathrm{~h}$. These exponential growth phase pre-cultures were centrifuged for 5 min at $4500 \mathrm{rpm}(3460.7 \mathrm{X} \mathrm{g})$ at $22^{\circ} \mathrm{C}$ and suspended in $2-3 \mathrm{~mL}$ of medium. Primary cultures were inoculated into $50 \mathrm{~mL}$ pre-warmed $\mathrm{BHI}, \mathrm{BHI}+2 \mathrm{~g} / \mathrm{L}$ D-Glucose, or $\mathrm{BHI}+2 \mathrm{~g} / \mathrm{L}{ }^{13} \mathrm{C}$-Glucose in a $50 \mathrm{~mL}$ conical tube, at an absorbance at $600 \mathrm{~nm}\left(A_{600}\right)$ of 0.08 , and incubated at $37^{\circ} \mathrm{C}$. The cultures were mixed by inversion every 30 min prior to sampling. The $A_{600}$ and $\mathrm{pH}$ were recorded every $30 \mathrm{~min}$ for $5 \mathrm{~h}$.

\section{Determination of glucose and lactate concentrations in cultivation media}

Cultivation media were harvested hourly $(1 \mathrm{~mL})$ by centrifugation at $13,200 \mathrm{rpm}(16,168 \mathrm{x} \mathrm{g})$, for $5 \mathrm{~min}$ at $4{ }^{\circ} \mathrm{C}$. The cell-free media were transferred to $1.5 \mathrm{~mL}$ microcentrifuge tubes, flash frozen in liquid nitrogen, and stored at $-20^{\circ} \mathrm{C}$ until use. Glucose and lactate concentrations in the culture media were determined from three independent cultures with kits purchased from R-Biopharm (Glucose test kit, \#10716251035 and L-Lactic acid test kit, \#10139084035).

\section{Glyceraldehyde 3-phosphate dehydrogenase (GAPDH) activity assay}

Due to differences in growth, bacteria were cultivated in BHI for $1.5 \mathrm{~h}$ (strain 351) or $2.5 \mathrm{~h}$ (strain 351-D10) and harvested by centrifugation at $5000 \mathrm{rpm}(4272.5 \mathrm{x} \mathrm{g})$ for $5 \mathrm{~min}$ at $4{ }^{\circ} \mathrm{C}$. Supernatants were discarded and the cell pellets were suspended in $750 \mu \mathrm{L}$ GAPDH assay buffer (BioVision, Inc) and lysed in lysing matrix B tubes using a FastPrep instrument. The lysates were centrifuged at $13,200 \mathrm{rpm}(16,168 \mathrm{x} \mathrm{g})$, for $5 \mathrm{~min}$ at $4{ }^{\circ} \mathrm{C}$. Supernatants were transferred to pre-chilled $1.5 \mathrm{~mL}$ microcentrifuge tubes. GAPDH activity was determined from three independent cultures according to the manufacture's instruction. Protein concentrations were determined using the Modified Lowry Protein Assay (Fisher Scientific). The harvest times and corresponding $A_{600}$ units were different from those used in metabolomics experiments because excess glucose was not added to the cultivation medium for enzyme assays.

\section{NMR sample preparation}

The preferred process for performing ${ }^{13} \mathrm{C}$-glucose metabolomics experiments is to use a glucose-free medium and add labelled glucose at a defined concentration. Unfortunately, glucose-free $\mathrm{BHI}$ was unavailable. Adding 2 $\mathrm{g} / \mathrm{L}$ of ${ }^{13} \mathrm{C}$-glucose into $\mathrm{BHI}$ containing unlabeled glucose $(2 \mathrm{~g} / \mathrm{L})$ meant that only $50 \%$ of the glucose was labeled. This limitation required the doubling of the number of bacteria harvested. Harvest times were chosen based on $\mathrm{A}_{600}$ numbers that gave maximal biomass, when most of the glucose was catabolized, but not when the bacteria were in the stationary phase. For these reasons, bacteria were harvested at two different times when the bacterial biomass was equivalent. Bacteria cultivated in $\mathrm{BHI}+{ }^{13} \mathrm{C}$-glucose were grown for $2 \mathrm{~h}$ (351) or $2 \mathrm{~h} 45 \mathrm{~min}$ (351-D10) and harvested by centrifugation at $4500 \mathrm{rpm}(3460.7 \mathrm{x} \mathrm{g})$ for $5 \mathrm{~min}$ at $0{ }^{\circ} \mathrm{C}$. Bacterial cell pellets were washed with ice cold ultrapure $\mathrm{H}_{2} \mathrm{O}$ (Milli$\mathrm{Q})$ and quenched in liquid nitrogen. Cells were placed on ice to thaw for $5 \mathrm{~min}$ and the cell pellets were suspended in $1 \mathrm{~mL}$ of ice cold ultrapure $\mathrm{H}_{2} \mathrm{O}$. The $A_{600}$ of the bacterial suspension was determined and $60 A_{600}$ units were diluted into $1 \mathrm{~mL}$ of ultrapure $\mathrm{H}_{2} \mathrm{O}$. The samples were transferred to lysing matrix B tubes (MP Biomedicals) and lysed for $40 \mathrm{~s}$ using a FastPrep instrument at a speed setting of 6 , then placed on ice for $5 \mathrm{~min}$. The lysing matrix B tubes were centrifuged at 13,200 rpm $(16,168 \times \mathrm{g})$ for $5 \mathrm{~min}$ at $0{ }^{\circ} \mathrm{C}$, and the supernatants were transferred to $2 \mathrm{~mL}$ microcentrifuge tubes pre-cooled to 
$0{ }^{\circ} \mathrm{C}$. Ice-cold $\mathrm{H}_{2} \mathrm{O}(1 \mathrm{~mL})$ was added to each lysing matrix $\mathrm{B}$ tube, vortexed, and the samples were lysed a second time. The lysing matrix $\mathrm{B}$ tubes were centrifuged for $5 \mathrm{~min}$ at $0{ }^{\circ} \mathrm{C}$, and the supernatants were pooled. The pooled cell-free lysates were clarified by centrifugation at $13,200 \mathrm{rpm}$ for $1 \mathrm{~min}$ at $0{ }^{\circ} \mathrm{C}$. Approximately $1.3 \mathrm{~mL}$ of the cell-free lysate from each sample was transferred to a prechilled $1.5 \mathrm{~mL}$ microcentrifuge tube. $1 \mathrm{~mL}$ of each cell-free lysate was flash frozen in liquid nitrogen and stored at $80^{\circ} \mathrm{C}$ prior to lyophilization for NMR analysis. The remaining cell-free lysate, approximately $300 \mu \mathrm{L}$, was used to determine protein concentrations (for normalization) with a modified Lowry protein assay (Fisher Scientific). Lyophilized frozen cell-free lysates were, suspended in $190 \mu \mathrm{L}$ of $50 \mathrm{mM}$ phosphate buffer $(\mathrm{pH}=7.2$, uncorrected) in $\mathrm{D}_{2} \mathrm{O}$, and $50 \mu \mathrm{M}$ of 3-(trimethylsilyl)propionic$2,2,3,3-\mathrm{d}_{4}$ acid sodium salt (TMSP) was added as a chemical shift reference. The samples were centrifuged for 20 min at $13,200 \mathrm{rpm}$ and $0{ }^{\circ} \mathrm{C}$ to remove any remaining cell debris. The supernatants were transferred to $3 \mathrm{~mm}$ NMR tubes for data collection.

\section{NMR data collection and analysis}

NMR spectra were collected at $298 \mathrm{~K}$ using a Bruker AVANCE III-HD $700 \mathrm{MHz}$ spectrometer equipped with a $5 \mathrm{~mm}$ quadruple resonance QCI-P cryoprobe $\left({ }^{1} \mathrm{H},{ }^{13} \mathrm{C}\right.$, ${ }^{15} \mathrm{~N}$, and ${ }^{31} \mathrm{P}$ ) with $\mathrm{z}$-axis gradients. A SampleJet sample changer, an automatic tuning and matching accessory, and ICON-NMR software were used to automate the NMR data collection. The $1 \mathrm{D}^{1} \mathrm{H}$ NMR spectra were collected with $32 \mathrm{~K}$ data points, 128 scans, 16 dummy scans, a relaxation delay of $1.5 \mathrm{~s}$, and a spectral width of $11,160 \mathrm{~Hz}$. The $1 \mathrm{D}{ }^{1} \mathrm{H}$ NMR spectra were collected using an excitation sculpting pulse sequence to suppress the water resonance [23]. The $2 \mathrm{D}{ }^{1} \mathrm{H}-{ }^{13} \mathrm{C}$ HSQC spectra were collected using non-uniform sampling (NUS) at a $25 \%$ sampling sparsity [24]. The $2 \mathrm{D}^{1} \mathrm{H}-{ }^{13} \mathrm{C}$ HSQC spectra were acquired with 32 scans, 16 dummy scans, and a $1.5 \mathrm{~s}$ relaxation delay. The spectra were collected with 2 $\mathrm{K}$ data points and a spectral width of $11,160 \mathrm{~Hz}$ in the direct dimension, and $1 \mathrm{~K}$ data points and a spectral width of $29,059 \mathrm{~Hz}$ in the indirect dimension.

\section{NMR data processing and analysis}

Our MVAPACK software suite (http://bionmr.unl.edu/mvapack.php) was used to process and analyze the $1 \mathrm{D}{ }^{1} \mathrm{H}$ NMR spectra [25]. The $1 \mathrm{D}{ }^{1} \mathrm{H}$ NMR spectra were processed with a single zero-fill and a $0.3 \mathrm{~Hz}$ line-broadening, and then Fourier transformed and automatically phased-corrected [26]. The 1D ${ }^{1} \mathrm{H}$ NMR spectra were normalized with standard normal variate normalization, aligned using icoshift [27], and then referenced to the TMSP peak at $0 \mathrm{ppm}$. Noise regions were automatically removed from the spectra and the residual water signal (4.6-4.8 $\mathrm{ppm})$ was manually removed. The $1 \mathrm{D}$
${ }^{1} \mathrm{H}$ NMR spectra were binned using adaptive intelligent binning [28] and scaled using unit variance (UV) scaling. The resulting data matrix was then used to create a principle component analysis (PCA) model.

NMRpipe [29] was used to process the $2 \mathrm{D}{ }^{1} \mathrm{H}_{-}{ }^{13} \mathrm{C}$ HSQC data set and NMRViewJ [30] was used to analyze the spectra and assign the metabolites. The Human Metabolomic Database (HMDB) (http://www.hmdb.ca/) was used to assign metabolites by matching reference chemical shifts from the database to the experimental spectra using a peak-error tolerance of $0.08 \mathrm{ppm}$ and $0.25 \mathrm{ppm}$ for ${ }^{1} \mathrm{H}$ and ${ }^{13} \mathrm{C}$ chemical shifts, respectively [31]. A data matrix consisting of relative metabolite peak intensities (rows) and biological replicates (columns) was produced from the $2 \mathrm{D}{ }^{1} \mathrm{H}-{ }^{13} \mathrm{C}$ HSQC data set $[32,33]$. The data matrix was normalized using probabilistic quotient (PQ) normalization [32, 33]. BioCyc (https://biocyc.org) was used to identify the dysregulated $S$. mitis metabolic pathways based on the observed metabolite changes [34].

\section{Statistical analysis}

Growth profiles and assays were performed in triplicate. Statistical differences in growth profiles were assessed by ANOVA, where a $p \leq 0.01$ was defined as statistically significant. Statistical differences in assays were assessed by Student's t-test, where a $p \leq 0.05$ was defined as statistically significant.

A pair-wise Student's t-test was used to determine if strain-dependent alterations in metabolite concentrations were statistically significant. The $p$-values were corrected using the Benjamini-Hochberg procedure to minimize the false discovery rate for multiple hypothesis testing [35]. Metabolite concentration changes were determined to be statistically significant by an FDR corrected $p$-value $<0.05$. In order to visualize the differences between $S$. mitis strains, MetaboAnalyst (https://www.metaboanalyst.ca) was used to generate a heatmap with hierarchical clustering from the $2 \mathrm{D}{ }^{1} \mathrm{H}_{-}{ }^{13} \mathrm{C}$ HSQC NMR peak intensities [36]. The data matrix of NMR peak intensities was scaled along each row using standard normal variate (SNV).

\section{Supplementary information}

Supplementary information accompanies this paper at https://doi.org/10. 1186/s12866-020-01849-w.

Additional file 1: Figure S1. A semi-log plot of bacterial growth with supplemental glucose.

Additional file 2: Figure S2. Glucose depletion and lactate accumulation as a function of growth

Additional file 3: Figure S3. Representative $2 \mathrm{D}{ }^{1} \mathrm{H}^{13} \mathrm{C} H S Q C N M R$ spectra for S. mitis strains 351 and 351-D10

\section{Abbreviations}

IE: Infective endocarditis; NMR: Nuclear magnetic resonance; BHI: Brain heart infusion medium; GAPDH: Glyceraldehyde 3-phosphate dehydrogenase; HSQC: Heteronuclear single quantum coherence 


\section{Acknowledgements}

Not applicable.

\section{Authors' contributions}

NNM and ASB provided strains and technical advice for cultivating S. mitis. GAS, RP, JMR, and AP oversaw experimental design. AP and JMR cultivated the bacteria for metabolomic analysis and AP analyzed the NMR spectra in consultation with RP. JMR and SGG performed metabolite, growth, and enzymatic experiments, while the data were analyzed by JMR and GAS. GAS, RP, JMR, NNM, ASB, SGG, and AP contributed to writing and editing the manuscript. All authors read and approved the final manuscript.

\section{Funding}

This research was supported in part by a grant from the National Institutes of Health (NIAID) 5RO-1 Al130056 (to ASB), by funding from the Redox Biology Center (P30 GM103335, NIGMS), and the Nebraska Center for Integrated Biomolecular Communication (P20 GM113126, NIGMS), and an intramural grant (\#531604-01-01 to NMM) from the Lundquist Institute-Harbor UCLA Medical Center, Torrance, CA. The research was performed in facilities renovated with support from the National Institutes of Health (RR015468-01). The funding sources had no role in experimental design or execution.

\section{Availability of data and materials}

The NMR metabolomics data can be found at https://figshare.com/s/6a04 fdcb4ac4546db276. All other data generated or analyzed during this study are included in this published article.

\section{Ethics approval and consent to participate} Not applicable.

\section{Consent for publication}

Not applicable.

\section{Competing interests}

The authors declare that they have no competing interests.

\section{Author details}

'Department of Chemistry, University of Nebraska-Lincoln, Lincoln, NE 68588-0304, USA. ${ }^{2}$ School of Veterinary Medicine and Biomedical Sciences, University of Nebraska-Lincoln, Lincoln, NE 68588-0905, USA. ${ }^{3}$ Present address: Chemical Testing Program, Wyoming Department of Health, Cheyenne, Wyoming 82002, USA. ${ }^{4}$ Present address: Department of Biological Sciences, Emporia State University, Emporia, Kansas 66801, USA. ${ }^{5}$ Division of Infectious Diseases, The Lundquist Institute at Harbor-UCLA Medical Center, Torrance, California 90502, USA. ${ }^{6}$ David Geffen School of Medicine University of California Los Angeles, Los Angeles, California 90095, USA. ${ }^{7}$ Nebraska Center for Integrated Biomolecular Communication, University of Nebraska-Lincoln, Lincoln, NE 68588-0304, USA

Received: 14 January 2020 Accepted: 9 June 2020

Published online: 15 June 2020

\section{References}

1. Cabell CH, Jollis JG, Peterson GE, Corey GR, Anderson DJ, Sexton DJ, Woods CW, Reller LB, Ryan T, Fowler VG Jr. Changing patient characteristics and the effect on mortality in endocarditis. Arch Intern Med. 2002;162:90-4.

2. Murdoch DR, Corey GR, Hoen B, Miro JM, Fowler VG Jr, Bayer AS, Karchmer AW, Olaison L, Pappas PA, Moreillon P, et al. Clinical presentation, etiology, and outcome of infective endocarditis in the 21st century: the international collaboration on endocarditis-prospective cohort study. Arch Intern Med. 2009;169:463-73

3. van Prehn J, van Triest Ml, Altorf-van der Kuil W, van Dijk K, Dutch National AMRSSG. Third-generation cephalosporin and carbapenem resistance in Streptococcus mitis/oralis. Results from a nationwide registry in the Netherlands. Clin Microbiol Infect. 2019:25:518-20.

4. Pericas JM, Nathavitharana R, Garcia-de-la-Maria C, Falces C, Ambrosioni J, Almela M, Garcia-Gonzalez J, Quintana E, Marco F, Moreno A, et al. Endocarditis caused by highly penicillin-resistant viridans group streptococci: still room for vancomycin-based regimens. Antimicrob Agents Chemother. 2019;63:e00516-9.
5. van der Linden M, Otten J, Bergmann C, Latorre C, Linares J, Hakenbeck R. Insight into the diversity of penicillin-binding protein 2x alleles and mutations in viridans streptococci. Antimicrob Agents Chemother. 2017;61: e02646-16.

6. Safdar A, Rolston KV. Vancomycin tolerance, a potential mechanism for refractory gram-positive bacteremia observational study in patients with cancer. Cancer. 2006;106:1815-20.

7. Garcia-de-la-Maria C, Pericas JM, Del Rio A, Castaneda X, Vila-Farres X, Armero $Y$, Espinal PA, Cervera C, Soy D, Falces $C$, et al. Early in vitro and in vivo development of high-level daptomycin resistance is common in mitis group streptococci after exposure to daptomycin. Antimicrob Agents Chemother. 2013;57:2319-25

8. Mishra NN, Tran TT, Seepersaud R, Garcia-de-la-Maria C, Faull K, Yoon A Proctor R, Miro JM, Rybak MJ, Bayer AS, et al. Perturbations of phosphatidate cytidylyltransferase (CdsA) mediate daptomycin resistance in Streptococcus mitis/oralis by a novel mechanism. Antimicrob Agents Chemother. 2017;61: e02435-16.

9. Tran TT, Mishra NN, Seepersaud R, Diaz L, Rios R, Dinh AQ, Garcia-de-laMaria C, Rybak MJ, Miro JM, Shelburne SA, et al. Mutations in cdsA and pgsA correlate with daptomycin resistance in Streptococcus mitis and S. oralis. Antimicrob Agents Chemother. 2019;63:e01531-18.

10. Adams HM, Joyce LR, Guan Z, Akins RL, Palmer KL. Streptococcus mitis and S. oralis lack a requirement for $\mathrm{Cds} A$, the enzyme required for synthesis of major membrane phospholipids in bacteria. Antimicrob Agents Chemother. 2017;61:e02552-16.

11. Gaupp R, Lei S, Reed JM, Peisker H, Boyle-Vavra S, Bayer AS, Bischoff M, Herrmann M, Daum RS, Powers R, et al. Staphylococcus aureus metabolic adaptations during the transition from a daptomycin susceptible phenotype to a daptomycin non-susceptible phenotype. Antimicrob Agents Chemother. 2015:59:4226-38.

12. Linder L, Andersson C, Sund ML, Shockman GD. Protoplast formation and localization of enzymes in Streptococcus mitis. Infect Immun. 1983;40:114654.

13. Boyle-Vavra S, Jones M, Gourley BL, Holmes M, Ruf R, Balsam AR, Boulware DR, Kline S, Jawahir S, Devries A, et al. Comparative genome sequencing of an isogenic pair of USA800 clinical methicillin-resistant Staphylococcus aureus isolates obtained before and after daptomycin treatment failure. Antimicrob Agents Chemother. 2011;55:2018-25.

14. Friedman L, Alder JD, Silverman JA. Genetic changes that correlate with reduced susceptibility to daptomycin in Staphylococcus aureus. Antimicrob Agents Chemother. 2006;50:2137-45.

15. Julian K, Kosowska-Shick K, Whitener C, Roos M, Labischinski H, Rubio A Parent L, Ednie L, Koeth L, Bogdanovich T, et al. Characterization of a daptomycin-nonsusceptible vancomycin-intermediate Staphylococcus aureus strain in a patient with endocarditis. Antimicrob Agents Chemother. 2007; 51:3445-8.

16. Murthy MH, Olson ME, Wickert RW, Fey PD, Jalali Z. Daptomycin nonsusceptible meticillin-resistant Staphylococcus aureus USA 300 isolate. J Med Microbiol. 2008:57:1036-8.

17. Jones T, Yeaman MR, Sakoulas G, Yang SJ, Proctor RA, Sahl HG, Schrenzel J, Xiong YQ, Bayer AS. Failures in clinical treatment of Staphylococcus aureus infection with daptomycin are associated with alterations in surface charge, membrane phospholipid asymmetry, and drug binding. Antimicrob Agents Chemother. 2008;52:269-78.

18. Mishra NN, Yang SJ, Sawa A, Rubio A, Nast CC, Yeaman MR, Bayer AS. Analysis of cell membrane characteristics of in vitro-selected daptomycinresistant strains of methicillin-resistant Staphylococcus aureus. Antimicrob Agents Chemother. 2009;53:2312-8.

19. Richardson AR, Somerville GA, Sonenshein AL. Regulating the intersection of metabolism and pathogenesis in gram-positive Bacteria. Microbiol Spectrum. 2015;3. https://doi.org/10.1128/microbiolspec.MBP-0004-2014.

20. Somerville GA, Proctor RA. At the crossroads of bacterial metabolism and virulence factor synthesis in staphylococci. Microbiol Mol Biol Rev. 2009;73: 233-48.

21. Gardner SG, Marshall DD, Daum RS, Powers R, Somerville GA. Metabolic mitigation of Staphylococcus aureus vancomycin intermediate-level susceptibility. Antimicrob Agents Chemother. 2018;62:e01608-17.

22. Reed JM, Gardner SG, Mishra NN, Bayer AS, Somerville GA. Metabolic interventions for the prevention and treatment of daptomycin nonsusceptibility in Staphylococcus aureus. J Antimicrob Chemother. 2019;74: 2274-83. 
23. Simpson AJ, Brown SA. Purge NMR: effective and easy solvent suppression J Magn Reson. 2005;175:340-6

24. Worley B, Powers R. Deterministic multidimensional nonuniform gap sampling. J Magn Reson. 2015;261:19-26.

25. Worley B, Powers R. MVAPACK: a complete data handling package for NMR metabolomics. ACS Chem Biol. 2014;9:1138-44.

26. Siegel MM. The use of the modified simplex-method for automatic phase correction in Fourier-transform nuclear magnetic-resonance spectroscopy. Analytica Chimica Acta-Comp Tech Optimization. 1981;5:103-8.

27. Savorani F, Tomasi G. Engelsen SB: icoshift: a versatile tool for the rapid alignment of 1D NMR spectra. J Magn Reson. 2010;202:190-202.

28. De Meyer T, Sinnaeve D, Van Gasse B, Tsiporkova E, Rietzschel ER, De Buyzere ML, Gillebert TC, Bekaert S, Martins JC, Van Criekinge W. NMR-based characterization of metabolic alterations in hypertension using an adaptive, intelligent binning algorithm. Anal Chem. 2008:80:3783-90.

29. Delaglio F, Grzesiek S, Vuister GW, Zhu G, Pfeifer J, Bax A. NMRPipe: a multidimensional spectral processing system based on UNIX pipes. J Biomol NMR. 1995;6:277-93.

30. Johnson BA. Using NMRView to visualize and analyze the NMR spectra of macromolecules. Methods Mol Biol. 2004;278:313-52.

31. Wishart DS, Jewison T, Guo AC, Wilson M, Knox C, Liu Y, Djoumbou Y, Mandal R, Aziat F, Dong E, et al. HMDB 3.0--the human Metabolome database in 2013. Nucleic Acids Res. 2013;41:D801-7.

32. Dieterle F, Ross A, Schlotterbeck G, Senn H. Probabilistic quotient normalization as robust method to account for dilution of complex biological mixtures. Application in ${ }^{1} \mathrm{H}$ NMR metabonomics. Anal Chem. 2006;78:4281-90

33. Vu T, Riekeberg E, Qiu Y, Powers R. Comparing normalization methods and the impact of noise. Metabolomics. 2018;14:108.

34. Karp PD, Ouzounis CA, Moore-Kochlacs C, Goldovsky L, Kaipa P, Ahren D, Tsoka S, Darzentas N, Kunin V, Lopez-Bigas N. Expansion of the BioCyc collection of pathway/genome databases to 160 genomes. Nucleic Acids Res. 2005;33:6083-9.

35. Benjamini $Y$, Hochberg $Y$. Controlling the false discovery rate: a practical and powerful approach to multiple testing. J R Stat Soc Ser B Methodol. 1995;57:289-300

36. Chong J, Yamamoto M, Xia J. MetaboAnalystR 2.0: From Raw Spectra to Biological Insights. Metabolites. 2019;9:e57.

\section{Publisher's Note}

Springer Nature remains neutral with regard to jurisdictional claims in published maps and institutional affiliations.

Ready to submit your research? Choose BMC and benefit from:

- fast, convenient online submission

- thorough peer review by experienced researchers in your field

- rapid publication on acceptance

- support for research data, including large and complex data types

- gold Open Access which fosters wider collaboration and increased citations

- maximum visibility for your research: over $100 \mathrm{M}$ website views per year

At $\mathrm{BMC}$, research is always in progress.

Learn more biomedcentral.com/submissions 